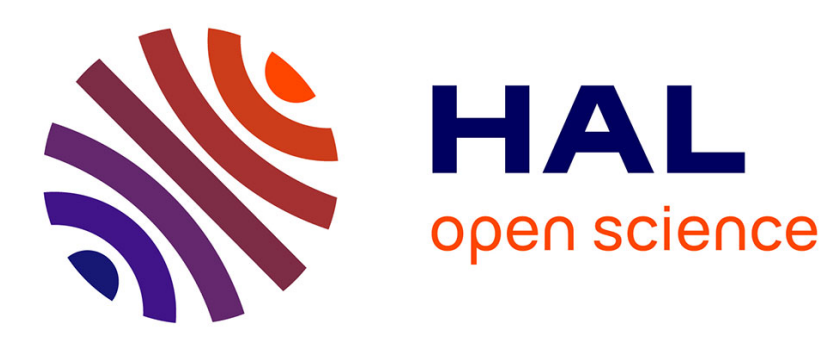

\title{
Global Asymptotic Stabilization for Nonminimum Phase Nonlinear Systems Admitting a Strict Normal Form
}

\author{
Vincent Andrieu, Laurent Praly
}

\section{To cite this version:}

Vincent Andrieu, Laurent Praly. Global Asymptotic Stabilization for Nonminimum Phase Nonlinear Systems Admitting a Strict Normal Form. IEEE Transactions on Automatic Control, 2008, 53 (5), pp.1120-1132. 10.1109/TAC.2008.923657 . hal-00362739

\section{HAL Id: hal-00362739 \\ https://hal.science/hal-00362739}

Submitted on 19 Feb 2009

HAL is a multi-disciplinary open access archive for the deposit and dissemination of scientific research documents, whether they are published or not. The documents may come from teaching and research institutions in France or abroad, or from public or private research centers.
L'archive ouverte pluridisciplinaire HAL, est destinée au dépôt et à la diffusion de documents scientifiques de niveau recherche, publiés ou non, émanant des établissements d'enseignement et de recherche français ou étrangers, des laboratoires publics ou privés. 


\title{
Global asymptotic stabilization for non-minimum phase non linear systems admitting a strict normal form
}

\author{
V. Andrieu*and L. Praly ${ }^{\dagger}$
}

February 19, 2009

\begin{abstract}
We address the problem of global asymptotic stabilization by output feedback for non minimum phase non linear systems which admit a strict normal form. We assume the knowledge of an observer and, depending on its properties, we propose various approaches to design the control law. Each of these approaches needs a different stabilizability assumption on the inverse dynamics. In this way, within a unified framework, we recover and extend some already published results and we establish new ones.
\end{abstract}

\section{Introduction}

We address the problem of global asymptotic stabilization by output feedback for systems whose dynamics can be written in the following strict' normal form :

$$
\left\{\begin{aligned}
\dot{z} & =F\left(z, \xi_{1}\right), \\
\dot{\xi}_{1} & =\xi_{2} \\
\dot{\xi}_{2} & =\xi_{3} \\
& \vdots \\
\dot{\xi}_{n} & =f\left(z, \xi_{1}, \ldots, \xi_{n}\right)+g\left(\xi_{1}\right) u, \\
y & =\xi_{1}
\end{aligned}\right.
$$

with state $\left(z, \xi_{1}, \ldots, \ldots, \xi_{n}\right)$ where $z$ is in $\mathbb{R}^{m}$ and $\xi_{i}$ is in $\mathbb{R}$, and where the function $F$ is in $C^{n+1}$, the functions $f$ and $g$ are in $C^{1}$ and we have :

$$
\begin{gathered}
F(0,0)=0 \quad, \quad f(0,0, \ldots, 0)=0, \\
g\left(\xi_{1}\right)>0 \quad \forall \xi_{1} \in \mathbb{R} .
\end{gathered}
$$

\footnotetext{
${ }^{*}$ V. Andrieu is with LAAS-CNRS, University of Toulouse, 31077 Toulouse, France (vincent.andrieu@gmail.com)

${ }^{\dagger}$ L. Praly is with CAS - Ecole des Mines de Paris, 35, rue St-Honore, 77305 Fontainebleau CEDEX, France, Laurent.Praly@ensmp.fr

${ }^{1}$ Only $\xi_{1}$ is present in the expression of $\dot{z}$.
} 
Systems whose dynamics can be written in the form (何) have been fully characterized, upon input scaling, by a coordinate free condition by Byrnes and Isidori in [9, Corollary 5.7]. It is trivially satisfied by any system whose dynamics can be written as :

$$
\left\{\begin{aligned}
\dot{y} & =b_{1}(y)+a_{1}(y) y_{2}, \\
\dot{y}_{2}= & b_{2}\left(y, y_{2}\right)+a_{2}(y) y_{3}, \\
& \vdots \\
\dot{y}_{n-1}= & b_{n-1}\left(y, y_{2}, \ldots, y_{n-1}\right)+a_{n-1}(y) y_{n}, \\
\dot{y}_{n}= & b_{n}\left(y, y_{2}, \ldots, y_{n}\right)+c_{0}(y) z_{1}+a_{n}(y) u \\
\dot{z}_{1}= & f_{1}\left(y, z_{1}\right)+c_{1}\left(y, z_{1}\right) z_{2}, \\
\vdots & \\
\dot{z}_{m-1}= & f_{m-1}\left(y, z_{1}, \ldots, z_{m-1}\right) \\
& +c_{m-1}\left(y, z_{1}, \ldots, z_{m-1}\right) z_{m} \\
\dot{z}_{m}= & f_{m}\left(y, z_{1}, \ldots, z_{m}\right) \\
& +c_{m}\left(y, z_{1}, \ldots, z_{m}\right) y
\end{aligned}\right.
$$

where the $a_{i}$ 's and $c_{j}$ 's take positive values. This is one of the most general (nominal) form for which we know how to design a globally asymptotically stabilizing output feedback and whose study has been initiated by Kanellakopoulos, Kokotović and Morse in [16] and Marino and Tomei in [22. In these works, the problem has been solved by imposing some restriction on the non-linearities (in [16, 22] the $b_{j}$ 's in (2) depend only of $y$ ) and by assuming that the $z$ dynamics, the inverse dynamics, are linear in $z$ and with an asymptotic stability property the minimum phase assumption -. From these original publications, many other results have been obtained, relaxing more and more the restriction on the functions $b_{j}$ 's but, for most of them, still preserving the minimum-phase assumption (see for instance [30, 28, 18, 31, 13, 2] and references therein).

Until recently the only significant results concerning non-minimum phase nonlinear systems were about semi-global stability, invoking high gain observers (see [8, 33 for instance). But fortunately, the minimum-phase assumption in the global stability case has been relaxed now, in particular by Karagiannis, Jiang, Ortega and Astolfi in [17, Marino and Tomei in [21] and by ourselves in the preliminary version [3] of this paper. In these contributions, the authors replace the minimum phase assumption by some specific form of state-stabilizability of the inverse dynamics. In other words, they assume (explicitly in [17, 3] and implicitly in [21]) the existence of a function $\phi_{z}$ such that the origin of the following system :

$$
\dot{z}=F\left(z, \phi_{z}(z)\right)
$$

is globally asymptotically stable. In [3], it is shown that, up to a regularity assumption, the existence of $\phi_{z}$ is necessary for the solvability of the output feedback stabilization problem for the system (11). Actually, more is required in [17, 21, 3]. Not only the origin should be asymptotically stable for (3) but this should be in a robust way with respect to disturbances which may act differently, depending on the context.

The unifying formalism we propose here allows us to rephrase and/or obtain output feedback stabilizers for the system (I) without a minimum-phase assumption and under various sets of assumptions. In Section 2, by exploiting a result of Freeman and Kokotović [11, we obtain a new result by following what we call the state disturbance or direct approach. In this case, the assumption is, in spirit, about the Input-to-State Stability (ISS) property of the 
following auxiliary system :

$$
\dot{z}=F\left(z, \phi_{z}\left(z+d_{z}\right)+d_{u}\right)
$$

where the disturbances $d_{z}$ and $d_{u}$ act as measurement error and input disturbance respectively. In Section 3, another result (encompassing the ones by Marino and Tomei [21] and Andrieu and Praly [3]) is obtained by following the dynamics error or indirect approach. There, the assumption is on :

$$
\dot{z}=F\left(z, \phi_{z}(z)\right)+d
$$

where the disturbance $d$ acts externally. Finally, in Section 4 , we show how by combining the two previous approaches and relying on an assumption on the system (4), we can recover the result of Karagiannis, Jiang, Ortega and Astolfi [17 in the case with no disturbances (or actually when they are part of the known system). Section 5 is devoted to illustrating examples and Section 6 contains our conclusions.

Above, we have quoted only the references in very direct relation with our topic and in particular with the non minimum phase case. Many other results are available, among which the most recent ones approach the design of output feedback via domination where the dominant model is mainly a simple chain of integrators but for which the output feedback is designed to cope with large disturbances. For this, it incorporates dynamically updated high gain controller and observer as in [13, 18] for instance or terms of higher order as dictated by weighted homogeneity as in [2, 31] for instance.

In the following proofs and examples, we focus on the ideas and concepts. Instead we do not detail the computations in particular when they could become heavy without bringing anymore light on our topic.

\section{The State Disturbance or Direct Approach}

\subsection{The context}

The popular "separation principle" is not true in general for global asymptotic stabilization. Nevertheless the following separation recipe is appropriate :

If we have:

1. An observer that provides boundedness and asymptotic convergence to zero of the state estimation error, independently of the control;

2. A state feedback that renders the system ISS with respect to additive error in its argument;

Then we can cook up a globally asymptotically stable output feedback.

This recipe, which may not have been written per se before, has already been followed by several authors (see for instance [6, 10, 34, 31]).

The first step in following this recipe is to introduce a state observer. For this, we rewrite the dynamics of the system (11) in other coordinates. Given $2(n-1)$ arbitrary but sufficiently 
differentiable functions $\left(a_{i}\right)_{1 \leq i \leq n-1}$, which take positive values, and $\left(b_{i}\right)_{1 \leq i \leq n-1}$, there exist two other functions $a_{n}$, which takes positive values, and $b_{n}$, and a diffeomorphism :

$$
\left(z, \xi_{1}, \ldots, \xi_{n}\right)^{T} \mapsto\left(z, y, \ldots, y_{n}\right)^{T}
$$

such that the dynamics of the system (1) can be rewritten in :

$$
\left\{\begin{aligned}
\dot{z} & =F(z, y) \\
\dot{y} & =a_{1}(z, y) y_{2}+b_{1}(z, y) \\
\dot{y}_{2}= & a_{2}\left(z, y, y_{2}\right) y_{3}+b_{2}\left(z, y, y_{2}\right) \\
\vdots & \\
\dot{y}_{n-1}= & a_{n-1}\left(z, y, y_{2}, \ldots, y_{n-1}\right) y_{n} \\
& +b_{n-1}\left(y, y_{2}, \ldots, y_{n-1}\right) \\
\dot{y}_{n}= & a_{n}(y) u+b_{n}\left(z, y, y_{2}, \ldots, y_{n}\right) .
\end{aligned}\right.
$$

We insist here for having $a_{n}$ to depend only on $y$.

With collecting $z$ and $y_{2}$ to $y_{n}$ into a single state vector $\mathcal{x}$ in $\mathbb{R}^{n+m-1}$, the dynamics (6) take the following form :

$$
\left\{\begin{array}{l}
\dot{x}=A(\mathcal{x}, y)+B(y) u \\
\dot{y}=C(\mathcal{x}, y)
\end{array}\right.
$$

Our detectability assumption is expressed as follows :

Assumption SD-D (State Disturbance, Detectability] :) The coordinates for $z$ and the functions $\left(a_{i}\right)_{1 \leq i \leq n-1}$, and $\left(b_{i}\right)_{1 \leq i \leq n-1}$ can be chosen in such a way that there exist a $C^{n+1}$ function $K: \mathbb{R} \rightarrow \mathbb{R}^{n+m-1}$ of $y$ and a positive definite symmetric matrix $P$ satisfying :

$P \frac{\partial A-K C}{\partial \mathcal{X}}(\mathcal{X}, y)+\frac{\partial A-K C}{\partial \mathcal{X}}(\mathcal{X}, y)^{T} P<0$

$$
\forall(\mathcal{X}, y) \in \mathbb{R}^{n+m-1} \times \mathbb{R}
$$

This assumption is discussed in the next subsection.

Following the recipe, the second step is to find a state feedback $\phi: \mathbb{R}^{n+m} \rightarrow \mathbb{R}$ such that the system :

$$
\left\{\begin{array}{l}
\dot{\mathcal{X}}=A(\mathcal{X}, y)+B(y) \phi(\mathcal{X}+e, y) \\
\dot{y}=C(\mathcal{X}, y)
\end{array}\right.
$$

is ISS with input $e$ in $\mathbb{R}^{n+m-1}$. As far as we know, this problem has not been solved for systems of the form (6). However, Freeman and Kokotović have given a solution in [1] for the particular case where the $z$-dynamics can also be written in a strict feedback form. Specifically the appropriate assumption is :

Assumption SD-S (State Disturbance, Stabilizability) : The z dynamics have a strict feedback form, i.e. they are:

$$
\left\{\begin{array}{c}
\dot{z}_{1}=f_{1}\left(z_{1}\right)+c_{1}\left(z_{1}\right) z_{2} \\
\vdots \\
\dot{z}_{m-1}=f_{m-1}\left(z_{1}, \ldots, z_{m-1}\right) \\
\quad+c_{m-1}\left(z_{1}, \ldots, z_{m-1}\right) z_{m} \\
\dot{z}_{m}=f_{m}\left(z_{1}, \ldots, z_{m}\right)+c_{m}\left(z_{1}, \ldots, z_{m}\right) y
\end{array}\right.
$$


where the functions $c_{j}$ take strictly positive values.

Designing a globally stabilizing output feedback under assumptions SD-D and SD-S is an easy task in principle by following the procedure proposed by Freeman and Kokotović in [11] and by invoking the ISS formalism. Precisely, we have :

Theorem 1 (State Disturbance approach) If the assumptions $S D$-D and $S D$-S hold then there exists a globally stabilizing dynamic output feedback of dimension $m+n-1$.

Proof : With assumption SD-S, the dynamics of the system (6) have a strict feedback form. Thus we can apply the design given in [11] to get a $C^{1}$ function $\phi: \mathbb{R}^{n+m} \rightarrow \mathbb{R}$ and a $C^{1}$, positive definite and proper function $V: \mathbb{R}^{n+m} \rightarrow \mathbb{R}_{+}$such that along the solutions of the system (9) we get, for some function $\gamma$ of class $\mathcal{K}_{\infty}$ and for all $(\mathcal{X}, y)$ in $\mathbb{R}^{n+m}$ and $e$ in $\mathbb{R}^{n+m-1}$, $\frac{\partial V}{\partial \mathcal{X}}(\mathcal{X}, y)[A(\mathcal{X}, y)+B(y) \phi(\mathcal{X}-e, y)]$

$$
+\frac{\partial V}{\partial y}(\mathcal{X}, y) C(\mathcal{X}, y) \leq-V(\mathcal{X}, y)+\gamma(|e|) .
$$

The output feedback is then defined as $u=\phi(\widehat{\mathcal{X}}, y)$ where $\widehat{\mathcal{X}}=\left(\hat{z}, \hat{y}_{2}, \ldots, \hat{y}_{n}\right)$ is given by the following (reduced order) observer :

$$
\left\{\begin{array}{l}
\widehat{\mathcal{x}}=w+M(y) \\
\dot{w}=A(\widehat{\mathcal{X}}, y)+B(y) u-K(y) C(\widehat{\mathcal{X}}, y)
\end{array}\right.
$$

with $M(y)=\int_{0}^{y} K(s) d s$.

With this feedback, the dynamics of the closed loop system can be written as :

$$
\left\{\begin{aligned}
\dot{x}= & A(\mathcal{X}, y)+B(y) \phi(\mathcal{x}-e, y) \\
\dot{y}= & C(\mathcal{X}, y) \\
\dot{e}= & A(\mathcal{X}, y)-A(\mathcal{X}-e, y) \\
& \quad-K(y)[C(\mathcal{X}, y)-C(\mathcal{X}-e, y)]
\end{aligned}\right.
$$

where $e=\mathcal{X}-\widehat{\mathcal{X}}$. It is seen as the interconnection of the system to be controlled and the error system. We have :

$$
\begin{gathered}
A(\mathcal{X}, y)-A(\mathcal{x}-e, y) \\
-K(y)[C(\mathcal{X}, y)-C(\mathcal{X}-e, y)] \\
=\left[\int_{0}^{1} \frac{\partial A-K C}{\partial \mathcal{X}}(\mathcal{X}+(1-s) e, y) d s\right] e .
\end{gathered}
$$

So, with (8), to any compact subset $\mathcal{C}$ of $\mathbb{R}^{2(n+m)-1}$, we can associate a strictly positive real number $c$ satisfying :

$$
\overbrace{e^{T} P e} \leq-c e^{T} P e \quad \forall(\mathcal{X}, y, e) \in \mathcal{C} .
$$

Inequalities (15) and (11) imply successively that, along the solutions of the closed loop system, $|e|$ and $V(\mathcal{X}, y)$ are bounded. Specifically, we get, for all $t \geq 0$,

$$
\begin{aligned}
e(t)^{T} P e(t) & \leq e(0)^{T} P e(0), \\
V(\mathcal{X}(t), y(t)) & \leq V(\mathcal{X}(0), y(0))+\gamma\left(\frac{e(0)^{T} P e(0)}{\lambda_{\min }(P)}\right),
\end{aligned}
$$


where $\lambda_{\min }(P)$ is the smallest eigenvalue of $P$ and the argument $t$ represents the time for the evaluation of the argument of the functions along the solution. These inequalities imply the global stability of the origin. Therefore, with (15), for each initial condition, there exists a strictly positive real number $c$ such that :

$$
|e(t)| \leq \exp (-c t)|e(0)| \sqrt{\frac{\lambda_{\max }(P)}{\lambda_{\min }(P)}}
$$

But, with the variation of constant formula and by splitting the integration interval $[0, t]$ in $\left[0, \frac{t}{2}\right] \cup\left(\frac{t}{2}, t\right],(11)$ gives, still for any solution and for any $t \geq 0$,

$V(\mathcal{X}(t), y(t)) \leq \exp (-t) V(\mathcal{x}(0), y(0))+$

$$
\exp \left(-\frac{t}{2}\right) \sup _{0 \leq s \leq \frac{t}{2}} \gamma(|e(s)|)+\sup _{\frac{t}{2} \leq s} \gamma(|e(s)|) .
$$

With (16), this implies that $V(\mathcal{x}(t), y(t))$ and therefore $\mathcal{x}(t)$ and $y(t)$ converge to 0 as $t$ goes to infinity. This establishes the global attractivity of the origin.

\subsection{Discussion}

\subsubsection{On the Detectability Assumption SD-D}

Guaranteeing the existence of a reduced order observer from assumption SD-D is a triviality. We have given ourselves this derogation in writing this assumption since sufficient conditions for it to hold are known. Specifically :

- Monotonic non linearities : Following Arcak and Kokotović [5], consider the case where we can find a function $K$ leading to the following decomposition :

$$
A(\mathcal{X}, y)-K(y) C(\mathcal{X}, y)=F \mathcal{X}+Q(y)
$$

$$
+\sum_{i=1}^{n+m-1} G_{i} \gamma_{i}\left(L_{i}^{T} \mathcal{X}, y\right)
$$

where, $\forall(s, y) \in \mathbb{R}^{2}$,

$$
-\infty<a_{i} \leq \frac{\partial \gamma_{i}}{\partial s}(s, y) \leq b_{i} \leq+\infty
$$

Proposition 1 ([5]) In this context, the inequality (8) holds if there exists a positive definite matrix symmetric $P$ and real numbers $\lambda_{i} \neq 0$ satisfying: $\sum_{i=1}^{n+m-1} \frac{b_{i}}{4}\left|\lambda_{i} L_{i}+\frac{P}{\lambda_{i}} G_{i}\right|^{2}$

$$
-\sum_{i=1}^{n+m-1} \frac{a_{i}}{4}\left|\lambda_{i} L_{i}-\frac{P}{\lambda_{i}} G_{i}\right|^{2}
$$

$$
+P F+F^{T} P \leq-I
$$

- Output dependent incremental rate : Following Krishnamurthy and Khorrami [19], we consider systems admitting the representation (2). To simplify our presentation, we 
introduce the functions $\phi_{i, j}$ and $\psi_{i}$ as :

$$
\begin{aligned}
& \phi_{i, j}= \begin{cases}\frac{\partial b_{i}}{\partial y_{j}} & 2 \leq j \leq i \leq n \\
0 & n+1 \leq i \leq m, 2 \leq j \leq n \\
\frac{\partial f_{i-n}}{\partial z_{j-n}} & n+1 \leq j \leq i \leq n+m\end{cases} \\
& \psi_{i}= \begin{cases}a_{i} & 2 \leq i \leq n-1 \\
c_{i-n} & n \leq i \leq n+m-1\end{cases}
\end{aligned}
$$

Proposition 2 ( [19, Theorem 2] ) If there exists a positive real number $\rho$, such that, for all $(\mathcal{X}, y)$ in $\mathbb{R}^{n+m}$, we have :

$$
\begin{aligned}
\rho & \leq \psi_{i}, 2 \leq i \leq n+m-1, \\
\rho \psi_{i} & \leq \psi_{i-1}, 3 \leq i \leq n+m-1, \\
\rho\left|\phi_{i, j}\right| & \leq \sqrt{\psi_{i} \psi_{j-1}}, 3 \leq i \leq n+m-1, \\
\rho\left|\phi_{n+m, j}\right| & \leq \sqrt{\psi_{n+m-1} \psi_{j-1}}, 3 \leq j \leq i,
\end{aligned}
$$

then there exists a continuous function $K$ and a matrix $P$ such that (8) is satisfied.

Another point about the detectability assumption SD-D is that, very often, the degree of freedom left in the definition of the functions $a_{i}$ 's and $b_{i}$ 's is forgotten in the literature. To illustrate it, consider the following second order system with no inverse dynamics :

$$
\left\{\begin{array}{l}
\dot{\xi}_{1}=\xi_{2} \\
\dot{\xi}_{2}=f\left(\xi_{1}, \xi_{2}\right)+g\left(\xi_{1}\right) u, \\
y=\xi_{1}
\end{array}\right.
$$

Then the functions $a_{1}, a_{2}, b_{1}$ and $b_{2}$ are free up to satisfying the following constraints:

$g(y)=a_{1}(y) a_{2}(y)>0$,

$f\left(y, a_{1}(y) y_{2}+b_{1}(y)\right)=$

$$
\left[a_{1}^{\prime}(y) y_{2}+b_{1}^{\prime}(y)\right]\left[a_{1}(y) y_{2}+b_{1}(y)\right]
$$

$$
+a_{1}(y) b_{2}\left(y, y_{2}\right)
$$

It follows that (8) holds if we can find a positive $C^{1}$ function $a_{1}$ and a $C^{1}$ function $\ell$ such that we have :

$$
\frac{\partial f}{\partial \xi_{2}}\left(\xi_{1}, \xi_{2}\right)<\ell\left(\xi_{1}\right)+2 \frac{a_{1}^{\prime}\left(\xi_{1}\right)}{a_{1}\left(\xi_{1}\right)} \xi_{2} \quad \forall\left(\xi_{1}, \xi_{2}\right) .
$$

Indeed in this case, we pick :

$$
\begin{aligned}
a_{2}(y) & =\frac{g(y)}{a_{1}(y)}, \quad b_{1}(y)=0 \\
b_{2}\left(y, y_{2}\right) & =\frac{f\left(y, a_{1}(y) y_{2}\right)-a_{1}(y) a_{1}^{\prime}(y) y_{2}^{2}}{a_{1}(y)}
\end{aligned}
$$

and (8) holds with :

$$
P=1 \quad, \quad k_{2}(y)=\frac{\ell(y)}{a_{1}(y)}
$$




\subsubsection{On the Stabilizability Assumption SD-S}

The specific strict feedback form imposed on the $z$-dynamics by the stabilizability assumption SD-S implies the existence of a function $\phi_{z}$ such that the origin of (3) is globally asymptotically stable. We have mentioned in the introduction that the existence of $\phi_{z}$ is "almost" necessary. So the main restriction imposed by assumption SD-S is the fact that, as proved by Freeman and Kokotović [11, it allows us to get a function $\phi_{z}$ which not only stabilizes asymptotically the origin of the $z$-subsystem but also insures the ISS property of the following auxiliary system :

$$
\dot{z}=F\left(z, \phi_{z}\left(z+d_{z}\right)+d_{u}\right)
$$

with input $\left(d_{z}, d_{u}\right)$ (see assumption SD-S' in section (1). It would be very useful to know whether or not, assumption SD-S can be replaced by this ISS property, i.e. whether or not the recursive Lyapunov design of [11] applies or can be modified to get $V$ and $\phi$ satisfying (11).

\section{The Dynamics Error or Indirect Approach}

This section is a reproduction of our conference paper [3].

\subsection{The context}

Another usual approach to design an output feedback is again to design the observer first but then to design the state feedback for this observer and not for the system to be controlled as done in the previous section. Specifically the state feedback is designed for the following system with state $(\widehat{\mathcal{X}}, y)$ given by the observer (12) :

$$
\left\{\begin{array}{l}
\dot{y}=C(\hat{\mathcal{x}}, y)+\Delta C \\
\dot{\hat{x}}=A(\widehat{\mathcal{x}}, y)+B(y) u+K(y) \Delta C
\end{array}\right.
$$

where, the term $\Delta C=C(\mathcal{X}, y)-C(\hat{\mathcal{X}}, y)$ is the correction term. Despite, this term is a good term for the observer, it is considered as a disturbance in the design of the state feedback. This approach that we call the dynamics error or indirect approach, is therefore the application of another separation recipe :

If we have :

1. An observer providing $L^{2}$-correction terms;

2. A state feedback making the system $L^{2}-I S S$;

Then we can cook up a globally asymptotically stable output feedback law.

Again this recipe may not have been formalized in this way previously (see however 4 , 29]) but it is certainly not new. Most of the published results on output feedback stabilization, starting from [16, 22], can be reinterpreted along its lines (see for instance [30, 28, 13, 21, 2]). To follow this recipe we propose the following set of assumptions :

\section{Assumption DE-D2 (Dynamics Error, $L^{2}$-Detectability) :}

DE-D2.1 The coordinates for $z$ and the functions $\left(a_{i}\right)_{1 \leq i \leq n-1}$ and $\left(b_{i}\right)_{1 \leq i \leq n-1}$ can be chosen in such a way that there exist a $C^{n+1}$ function $K$ of $y$ and a positive definite symmetric matrix $P$ satisfying 
$P \frac{\partial A-K C}{\partial \mathcal{X}}(\mathcal{X}, y)+\frac{\partial A-K C}{\partial \mathcal{X}}(\mathcal{X}, y)^{T} P$

$$
\leq-\frac{\partial C}{\partial \mathcal{X}}(\mathcal{x}, y)^{T} \frac{\partial C}{\partial \mathcal{X}}(\mathcal{x}, y)
$$$$
\forall(\mathcal{X}, y) \in \mathbb{R}^{n+m-1} \times \mathbb{R}
$$

DE-D2.2 The system (1) is zero-state detectable, i.e. any solution $\mathcal{X}(\mathcal{X}, t)$ of :

$$
\dot{\mathcal{X}}=A(\mathcal{X}, 0) \quad, \quad C(\mathcal{X}, 0)=0
$$

is defined on $[0,+\infty)$ and converges to 0 as $t$ tends to infinity.

Assumption DE-S2 (Dynamics Error, $L^{2}$-Stabilizability) : There exists a $C^{n+1}$ function $\phi_{z}$, zero at the origin, and such that the following system is $L^{2}$-ISS :

$$
\dot{z}=F\left(z, \phi_{z}(z)\right)+K_{z}\left(\phi_{z}(z)\right) d
$$

where $K_{z}$ collects all the $z$-components of the function $K$. Specifically, there exist a $C^{n+1}$, positive definite and proper function $V_{z}$ and a positive definite continuous function $\alpha_{z}$ such that we have:

$\frac{\partial V_{z}}{\partial z}(z)\left[F\left(z, \phi_{z}(z)\right)+K_{z}\left(\phi_{z}(z)\right) d\right]$

$$
\leq-\alpha_{z}(z)+|d|^{2}
$$

These two assumptions are discussed in the next subsection.

Again, designing a globally stabilizing output feedback under this set of assumptions is an easy task by relying on the observer backstepping technique. Precisely, we have :

Theorem 2 (Dynamics Error, $L^{2}$ case) If the assumptions DE-D2 and DE-S2 hold then there exists a globally stabilizing dynamic output feedback of dimension $m+n-1$.

Proof : Consider again the observer (12). We have :

$|C(\widehat{\mathcal{x}}, y)-C(\mathcal{x}, y)|^{2}$

$$
\begin{aligned}
& =\left|\left[\int_{0}^{1} \frac{\partial C}{\partial \mathcal{X}}(\mathcal{X}+s[\widehat{\mathcal{X}}-\mathcal{X}], y) d s\right][\widehat{\mathcal{X}}-\mathcal{X}]\right|^{2}, \\
& \leq \int_{0}^{1}\left|\left[\frac{\partial C}{\partial \mathcal{X}}(\mathcal{X}+s[\widehat{\mathcal{X}}-\mathcal{x}], y)\right][\widehat{\mathcal{X}}-\mathcal{X}]\right|^{2} d s .
\end{aligned}
$$

So, with (14) and (23), we get :

$$
\begin{aligned}
\overparen{(\widehat{\mathcal{X}}-\mathcal{X})^{T} P(\widehat{\mathcal{X}}-\mathcal{X})} & \leq-|C(\widehat{\mathcal{X}}, y)-C(\mathcal{X}, y)|^{2} \\
& =-|\Delta C|^{2} .
\end{aligned}
$$

This establishes that the observer makes the correction term $\Delta C$ an $L^{2}$ function along the solutions of the closed loop system. So, according to the above separation recipe, it remains 
to design a state feedback making $L^{2}$-ISS the following system with input $d$ :

$$
\left\{\begin{array}{l}
\dot{\hat{z}}=F(\hat{z}, y)+K_{z}(y) d \\
\dot{y}=a_{1}(\hat{z}, y) \hat{y}_{2}+b_{1}(\hat{z}, y)+d \\
\dot{\hat{y}}_{2}=a_{2}\left(\hat{z}, y, \hat{y}_{2}\right) \hat{y}_{3}+b_{2}\left(\hat{z}, y, \hat{y}_{2}\right)+K_{2}(y) d \\
\quad \vdots \\
\dot{\hat{y}}_{n}=a_{n}(y) u+b_{n}\left(\hat{z}, y, \hat{y}_{2}, \ldots, \hat{y}_{n}\right)+K_{n}(y) d .
\end{array}\right.
$$

Using assumption DE-S2, we have a $C^{n+1}$, positive definite and proper function $V_{z}$ satisfying :

$$
\frac{\partial V_{z}}{\partial \hat{z}}(\hat{z})\left(F\left(\hat{z}, \phi_{z}(\hat{z})\right)+K_{z}\left(\phi_{z}(\hat{z})\right) d\right) \leq-\alpha_{z}(\hat{z})+|d|^{2} .
$$

By applying recursively Lemma 1 given in the appendix, we can propagate this property up to getting a $C^{1}$, positive definite and proper function $V_{n}$ and a $C^{1}$ function $\phi_{n}$ such that $u=\phi_{n}\left(\hat{z}, y, \hat{y}_{2}, \ldots, \hat{y}_{n}\right)$ gives for the system (27) :

$\overbrace{V_{n}\left(\hat{z}, y, \hat{y}_{2}, \ldots, \hat{y}_{n}\right)} \leq-\alpha_{n}\left(\hat{z}, y, \hat{y}_{2}, \ldots, \hat{y}_{n}\right)+|d|^{2}$

where $\alpha_{n}$ is a positive definite continuous function.

So now, instead of viewing the dynamics of the closed loop system as the interconnection of the system to be controlled and the error system, as in the state disturbance approach (see (13)), we view them as the interconnection of the observer (27) with input

$$
d=\Delta C=C(e+\hat{x}, y)-C(\hat{x}, y)
$$

and the error system

$$
\dot{e}=A(\widehat{\mathcal{X}}+e, y)-A(\widehat{\mathcal{X}}, y)-K(y) \Delta C,
$$

with output $\triangle C$ and input $y$ and

$$
\widehat{\mathcal{x}}=\left(\hat{z}, \hat{y}_{2}, \ldots, \hat{y}_{n}\right)
$$

As proved above, the latter generates a function $\Delta C$ which is square-integrable along the solutions of the closed loop system and the former is $L^{2}$-ISS with this function as input. From here proving global asymptotic stability is easy. Indeed, with (26) and (28), we get readily :

$$
\overbrace{e^{T} P e+V_{n}(\hat{\mathcal{x}}, y)} \leq-\alpha_{n}(\hat{\mathcal{x}}, y)
$$

Since $\alpha_{n}$ is a positive definite continuous function of its arguments, this establishes global stability of the origin as well as the convergence of any solution to the largest invariant set contained in the set $\{(e, \widehat{\mathcal{x}}, y): \widehat{\mathcal{x}}=y=0\}$. In this set, we have

$$
\dot{\mathcal{X}}=A(\mathcal{X}, 0) \quad, \quad C(\mathcal{X}, 0)=0 .
$$

So, by following the same arguments as in [12, p.44], we can conclude with assumption DED2.2, that each solution converges to the origin, i.e., we have global attractivity. 


\subsection{Discussion}

\subsubsection{On Assumption DE-S2}

Again, the main restriction imposed by assumption DE-S2 is the fact that the function $\phi_{z}$ not only stabilizes asymptotically the origin of the $z$-subsystem but also that it provides the $L^{2}$-ISS property of the following auxiliary system :

$$
\dot{z}=F\left(z, \phi_{z}(z)\right)+d
$$

where the disturbance $d$ acts externally instead of internally as we had with the state disturbance approach (See (21)).

\subsubsection{On Assumption DE-D2}

Assumption DE-D2 is very similar to assumption SD-D. We have only replaced $<0$ in (8) by $\leq-\frac{\partial C}{\partial \mathcal{X}} \frac{\partial C}{\partial \mathcal{X}}$ in (23). However, even with strengthening (8) as

$$
P \frac{\partial A-K C}{\partial \mathcal{X}}(\mathcal{X}, y)+\frac{\partial A-K C}{\partial \mathcal{X}}(\mathcal{X}, y)^{T} P \leq-I
$$

$$
(\mathcal{X}, y) \in \mathbb{R}^{n+m-1} \times \mathbb{R},
$$

it is a difficult task to go from this stronger version of SD-D to DE-D2.1. For this we need an extra property on the function $C$. Typically it is that $\left|\frac{\partial C}{\partial \mathcal{X}}(\mathcal{X}, y)\right|$ is bounded or more specifically that $a_{1}$ does not depend on $z$ and $\left|\frac{\partial b_{1}}{\partial z}(z, y)\right|$ is bounded. Without such a property, a possibility is to redesign the observer by augmenting the gain $K$. This idea has been exploited already in the literature (see [14, 27, 7] for instance). Here it can be exploited at least in the case where $C$ is affine in $\mathcal{X}$, i.e.

$$
C(\mathcal{X}, y)=C_{0}(y)+C_{1}(y) \mathcal{X} .
$$

In this case (8) reads :

$$
\begin{aligned}
& {\left[P \frac{\partial A}{\partial \mathcal{X}}(\mathcal{X}, y)+\frac{\partial A}{\partial \mathcal{X}}(\mathcal{X}, y)^{T} P\right] } \\
&-\left[P K(y) C_{1}(y)+C_{1}(y)^{T} K(y)^{T} P\right]<0
\end{aligned}
$$$$
\forall(\mathcal{X}, y) \in \mathbb{R}^{n+m-1} \times \mathbb{R} .
$$

So, by augmenting $K(y)$ with $\frac{1}{2} P^{-1} C_{1}(y)^{T}$, we get :

$$
\begin{array}{r}
{\left[P \frac{\partial A}{\partial \mathcal{X}}(\mathcal{X}, y)+\frac{\partial A}{\partial \mathcal{X}}(\mathcal{X}, y)^{T} P\right]} \\
-\left[P\left(K(y)+\frac{1}{2} P^{-1} C_{1}(y)^{T}\right) C_{1}(y)\right. \\
<-C_{1}(y)^{T} C_{1}(y)=-\frac{\partial C}{\partial \mathcal{X}}(\mathcal{X}, y)^{T} \frac{\partial C}{\partial \mathcal{X}}(\mathcal{X}, y) \\
\quad \forall(\mathcal{X}, y) \in \mathbb{R}^{n+m-1} \times \mathbb{R}
\end{array}
$$


which is (23). For example, for the system (19), the assumption (23) still holds under the constraint (20). For this, it is sufficient to modify $k_{2}$ as :

$$
k_{2}(y)=\frac{\ell(y)}{a_{1}(y)}+\frac{a_{1}(y)}{2} .
$$

Another possibility of relaxing assumption DE-D2 is offered when the correction term can be decomposed as (see [29]) :

$$
C(\mathcal{x}, y)-C(\hat{\mathcal{x}}, y)=m(\hat{z}, y) \varepsilon(z, \hat{z}, y) .
$$

In this case it is sufficient that the observer ensures that the term $\varepsilon(z, \hat{z}, y)$ is in $L^{2}$ along the solutions. But then the stabilizability assumption DE-S2 is about the following system :

$$
\dot{z}=F\left(z, \phi_{z}(z)\right)+K_{z}\left(\phi_{z}(z)\right) m\left(\hat{z}, \phi_{z}(z)\right) d .
$$

This extension is used for the system (44) studied below.

If none of the above succeeds, we abandon the $L^{2}$ framework and try the following $L^{1}$ one. The assumptions we need then are (see also [29, 0]).

\section{Assumption DE-D1 (Dynamics Error, $L^{1}$-Detectability) :}

DE-D1.1 The coordinates for $z$ and the functions $\left(a_{i}\right)_{1 \leq i \leq n-1}$ and $\left(b_{i}\right)_{1 \leq i \leq n-1}$ can be chosen in such a way that there exist $a C^{n+1}$ function $K$ and a positive semi-definite symmetric matrix $P$ and $k$ vectors $v_{i}$ satisfying :

$$
P+\sum_{i=1}^{k} v_{i} v_{i}^{T}>0
$$

$\frac{e^{T} P \frac{\partial A-K C}{\partial \mathcal{X}}(\mathcal{X}, y) e}{\sqrt{e^{T} P e}}$

$$
\begin{aligned}
&+\sum_{i=1}^{k} \operatorname{sign}\left(v_{i}^{T} e\right) v_{i}^{T} \frac{\partial A-K C}{\partial \mathcal{X}}(\mathcal{X}, y) e \\
& \leq-\left|\frac{\partial C}{\partial \mathcal{X}}(\mathcal{X}, y) e\right| \\
& \forall(\mathcal{X}, y, e) \in \mathbb{R}^{n+m-1} \times \mathbb{R} \times \mathbb{R}^{n+m-1}
\end{aligned}
$$

Moreover the $K_{z}$ component of $K$ is bounded.

DE-D1.2 Same as assumption DE-D2.2

Assumption DE-S1 (Dynamics Error, $L^{1}$-Stabilizability) : There exist a $C^{n+1}$ function $\phi_{z}$, zero at the origin, and a $C^{n+1}$, positive definite and proper function $V_{z}$ and a positive definite continuous function $\alpha_{z}$ satisfying :

$$
\frac{\partial V_{z}}{\partial z}(z)\left[F\left(z, \phi_{z}(z)\right)+d\right] \leq-\alpha_{z}(z)+|d|
$$

Theorem 3 (Dynamics Error, $L^{1}$ case) If the assumptions DE-D1 and DE-S1 hold then there exists a globally stabilizing dynamic output feedback of dimension $m+n-1$.

\footnotetext{
${ }^{2}$ This is equivalent to writing that $V_{z}$ has a bounded gradient.
} 
Proof : For a locally Lipschitz function $W$ and a system $\dot{e}=f(e, \mathcal{X})$, we denote by $D^{+} W$, the Dini derivative of $W$ along the solutions of this system, i.e.

$$
D^{+} W(e)=\limsup _{t \searrow 0} \frac{W(e+t f(e, \mathcal{X}))-W(e)}{t} .
$$

With this notation, the proof follows exactly the same lines as the one of Theorem 2 .

Since we have :

$$
\begin{aligned}
|C(\mathcal{x}+e, y)-C(\mathcal{x}, y)| & =\left|\int_{0}^{1} \frac{\partial C}{\partial \mathcal{X}}(\mathcal{x}+s e, y) e d s\right| \\
& \leq \int_{0}^{1}\left|\frac{\partial C}{\partial \mathcal{X}}(\mathcal{X}+s e, y) e\right| d s
\end{aligned}
$$

with (14) and (33) and $e$ satisfying (29), we getp :

$$
D^{+}\left(\sqrt{e^{T} P e}+\sum_{i=1}^{k}\left|v_{i}^{T} e\right|\right) \leq-|\Delta C| .
$$

This establishes that the observer makes the correction term $\Delta C$ an $L^{1}$ function along the solutions of the closed loop system. Moreover, the function $K_{z}$ being bounded, the same integrability property holds for $K_{z} \Delta C$.

Then we follow exactly the same lines as the ones after (26), except that, to propagate the $L^{1}$-ISS property, we apply recursively Lemma 2 given in the appendix. In this way, we get a $C^{1}$, positive definite and proper function $V_{n}$ and a $C^{1}$ function $\phi_{n}$ such that $u=\phi_{n}\left(\hat{z}, y, \hat{y}_{2}, \ldots, \hat{y}_{n}\right)$ gives for the system (27) :

$$
\overbrace{V_{n}\left(\hat{z}, y, \hat{y}_{2}, \ldots, \hat{y}_{n}\right)} \leq-\alpha_{n}\left(\hat{z}, y, \hat{y}_{2}, \ldots, \hat{y}_{n}\right)+|\Delta C|
$$

where $\alpha_{n}$ is a positive definite continuous function.

This yields the following for the closed loop system :

$D^{+}\left(\sqrt{e^{T} P e}+\sum_{i=1}^{k}\left|v_{i}^{T} e\right|+V_{n}\left(\hat{z}, y, \hat{y}_{2}, \ldots, \hat{y}_{n}\right)\right)$

$$
\leq-\alpha_{n}\left(\hat{z}, y, \hat{y}_{2}, \ldots, \hat{y}_{n}\right)
$$

With (32) and [32, Theorems II.6.2 and VII.3.2], we conclude that we have global stability of the origin and convergence of any solution to the largest invariant set contained in the set $\{(e, \widehat{x}, y): \widehat{x}=y=0\}$. From this point, the proof is completed as the one of Theorem 2 .

We end this section by mentioning that the very specific structure of the system studied by Marino and Tomei in 21] is such that the assumptions invoked in that work imply that, for all $p \geq 1$, both $L^{p}$-detectability and $L^{p}$-stabilizability are satisfied and so in particular assumptions DE-D1, DE-D2, DE-S1 and DE-S2 (see [1, Example 4.2.3]).

\footnotetext{
${ }^{3}$ The use of polyhedral Lyapunov functions has a long history in control theory (see [26] and the references therein, for instance).
} 


\section{Combined approach}

\subsection{The context}

In this section, we rephrase part of the result obtained by Karagiannis, Jiang, Ortega and Astolfi in [17 by viewing its proof as a combination of the state disturbance and the dynamics error approaches.

In this context, the stabilizability assumption is given as the following :

Assumption SD-S' : There exists a $C^{n+1}$ function $\phi_{z}$ zero at the origin, and such that the following system is ISS :

$$
\dot{z}=F\left(z, \phi_{z}\left(z+d_{z}\right)+d_{u}\right) .
$$

Specifically, there exist a $C^{n+1}$, positive definite and proper function $V_{z}$ and two continuous non negative functions $\gamma_{1}$ and $\gamma_{2}$ which are zero at zero and such that we have:

$\frac{\partial V_{z}}{\partial z}(z)\left[F\left(z, \phi_{z}\left(z+d_{z}\right)+d_{u}\right)\right] \leq$

$$
-V_{z}(z)+\gamma_{1}\left(\left|d_{z}\right|\right)+\gamma_{2}\left(\left|d_{u}\right|\right) \text {. }
$$

Theorem 4 (Combined approach) If the assumptions DE-D2 and SD-S' hold then there exists a globally stabilizing dynamic output feedback of dimension $m+n-1$.

Proof : From (34) we get readily :

$\frac{\partial V_{z}}{\partial z}(z) F(z, y) \leq$

$$
-V_{z}(z)+\gamma_{1}(|z-\hat{z}|)+\gamma_{2}\left(\left|y-\phi_{z}(\hat{z})\right|\right) .
$$

This establishes that the $z$-subsystem is ISS with respect to $z-\hat{z}$ and $y-\phi_{z}(\hat{z})$. The observer takes care of making the disturbance $z-\hat{z}$ "small". It remains to design a state feedback taking care of the other disturbance $y-\phi_{z}(\hat{z})$. To do so, we consider the coordinate :

$$
\mu=y-\phi_{z}(\hat{z})
$$

and write its dynamics as :

$$
\left\{\begin{array}{c}
\dot{\mu}=a_{1}(\hat{z}, y) \hat{y}_{2}+b_{1}(\hat{z}, y)+d \\
\quad-\frac{\partial \phi_{z}}{\partial z}(\hat{z})\left[F(\hat{z}, y)+K_{z}(y) d\right], \\
\vdots \\
\dot{\hat{y}}_{n}=a_{n}(y) u+b_{n}\left(\hat{z}, \ldots, \hat{y}_{n}\right)+K_{n}(y) d,
\end{array}\right.
$$

where, $\hat{z}$ is considered as a measured exogenous input for which we know its time derivative satisfies :

$$
\dot{\hat{z}}=F\left(\hat{z}, \mu+\phi_{z}(\hat{z})\right)+K_{z}\left(\mu+\phi_{z}(\hat{z})\right) d .
$$

Here $d$ is a seen as a disturbance but is actually the correction term associated to the observer :

$$
d=C(\mathcal{x}, y)-C(\hat{\mathcal{x}}, y) .
$$


We start the design by observing that the function $\phi_{1}$ defined as :

$\phi_{1}(\hat{z}, \mu)=\frac{1}{a_{1}(\hat{z}, y)}\left[-2 \mu-\left(\frac{\partial \phi_{z}}{\partial z}(\hat{z}) K_{z}(y)\right)^{2} \mu\right.$

$$
\left.-b_{1}(\hat{z}, y)+\frac{\partial \phi_{z}}{\partial z}(\hat{z})(F(\hat{z}, y))\right]
$$

where $y=\mu+\phi_{z}(\hat{z})$, gives, for the $\mu$-subsystem,

$$
\stackrel{\dot{\mu^{2}}}{\dot{2}} \leq-\mu^{2}+2|d|^{2} \quad \forall\left(\hat{z}, \mu, \hat{y}_{2}\right): \hat{y}_{2}=\phi_{1}(\mu, \hat{z})
$$

This establishes an $L^{2}$-ISS property for this $\mu$-subsystem. Then, by applying recursively Lemma 11 of the appendix, we can propagate this $L^{2}$-ISS property to get a $C^{1}$ function $\phi_{n}$ and a $C^{1}$, positive definite and proper function $V_{n}$ such that $u=\phi_{n}\left(\hat{z}, \mu, \hat{y}_{2}, \ldots, \hat{y}_{n}\right)$ gives, for the system (36),

$$
\overbrace{V_{n}\left(\mu, \hat{y}_{2}, \ldots, \hat{y}_{n}\right)} \leq
$$

where, $\alpha_{n}$ is a positive definite continuous function.

Note that here the recursive procedure starts with $\mu$ (equivalent to $y$ ) instead of $\hat{z}$ as in the dynamics error approach (see $(27)$ ).

With all these preliminaries, we can write the dynamics of the closed loop system as :

$$
\left\{\begin{aligned}
& \dot{z}= F\left(z, \phi_{z}(\hat{z})+\mu\right) \\
& \dot{\mu}= a_{1}(\hat{z}, y) \hat{y}_{2}+b_{1}(\hat{z}, y)+d \\
& \quad \quad-\frac{\partial \phi_{z}}{\partial z}(\hat{z})\left[F(\hat{z}, y)+K_{z}(y) d\right], \\
& \dot{\hat{y}}_{n}= a_{n}(y) \phi_{n}\left(\hat{z}, \mu, \hat{y}_{2}, \ldots, \hat{y}_{n}\right) \\
& \quad \quad+b_{n}\left(\hat{z}, y, \hat{y}_{2}, \ldots, \hat{y}_{n}\right)+K_{n}(y) d, \\
& \dot{e}=A(\widehat{\mathcal{x}}+e, y)-A(\widehat{\mathcal{x}}, y)-K(y) d,
\end{aligned}\right.
$$

with (38), $\hat{z}=z-e_{z}, y=\mu+\phi_{z}(\hat{z})$ and :

$$
\begin{gathered}
\widehat{\mathcal{X}}=\left(\hat{z}, \hat{y}_{2}, \ldots, \hat{y}_{n}\right) \quad, \quad e=\mathcal{X}-\widehat{\mathcal{X}}, \\
K(y)=\left(K_{z}(y), K_{2}(y), \ldots, K_{n}(y)\right) .
\end{gathered}
$$

It is seen as the interconnection of the error system and a system which combines the $z$-part of the system to be controlled and the $\left(\mu, \hat{y}_{2}, \ldots, \hat{y}_{n}\right)$-part of the observer.

With (35), (39), (26), (34) and (37) we get along the solutions of this system (40) :

$\overbrace{2 e^{T} P e+V_{n}\left(\mu, \hat{y}_{2}, \ldots, \hat{y}_{n}\right)} \leq$

$$
-\alpha_{n}\left(V_{n}\left(\mu, \hat{y}_{2}, \ldots, \hat{y}_{n}\right)\right)-|d|^{2}
$$

$$
\begin{aligned}
& \overparen{V_{z}(z)} \leq-V_{z}(z)+\rho\left(2 e^{T} P e+V_{n}\left(\mu, \hat{y}_{2}, \ldots, \hat{y}_{n}\right)\right) \\
& \overparen{V_{z}(\hat{z})} \leq-V_{z}(\hat{z})+\gamma_{2}(|\mu|)+\mathfrak{k}(y, \hat{z})|d|
\end{aligned}
$$


where $\rho$ is any class $\mathcal{K}_{\infty}$ function and $\mathfrak{k}$ is the continuous function satisfying :

$$
\begin{aligned}
\rho(s) & \geq 2 \max \left\{\gamma_{1}\left(\left|\frac{1}{2} P^{-\frac{1}{2}}\right| \sqrt{s}\right),\right. \\
\mathfrak{k}(y, \hat{z}) & =\left|\frac{\partial V_{z}}{\partial z}(\hat{z}) K_{z}(y)\right| .
\end{aligned}
$$

Inequalities (41) and (42) imply successively that, along the solutions of the closed loop system, $2 e^{T} P e+V_{n}\left(\mu, \hat{y}_{2}, \ldots, \hat{y}_{n}\right)$ and $V_{z}(z)$ are bounded. Specifically, we get, for all $t \geq 0$,

$$
2 e(t)^{T} P e(t)+V_{n}\left(\mu(t), \hat{y}_{2}(t), \ldots, \hat{y}_{n}(t)\right) \leq
$$

$V_{z}(z(t)) \leq V_{z}(0)$

$$
\begin{array}{r}
2 e(0)^{T} P e(0)+V_{n}\left(\mu(0), \hat{y}_{2}(0), \ldots, \hat{y}_{n}(0)\right), \\
+\rho\left(2 e(0)^{T} P e(0)+V_{n}\left(\mu(0), \hat{y}_{2}(0), \ldots, \hat{y}_{n}(0)\right),\right.
\end{array}
$$

where the argument $t$ represents the times for the evaluation of the argument of the functions along the solution. These inequalities imply the global stability of the origin.

Actually since $V_{n}$ is positive definite and $\overbrace{2 e^{T} P e+V_{n}\left(\mu, \hat{y}_{2}, \ldots, \hat{y}_{n}\right)}$ is strictly negative if $V_{n}\left(\mu, \hat{y}_{2}, \ldots, \hat{y}_{n}\right)$ and $d$ are not zero, we have also :

$$
\lim _{t \rightarrow+\infty} \sup _{s \geq t}\left[|\mu(s)|+|d(s)|+\sum_{i=2}^{n}\left|\hat{y}_{i}(s)\right|\right]=0 .
$$

Also, as we have done from (11), inequality (43) gives, for any bounded solution, a real number $\overline{\mathfrak{k}}$, such that we have for all $t \geq 0$ :

$$
\begin{aligned}
V_{z}(\hat{z}(t)) \leq \exp (-t) V_{z}(\hat{z}(0)) & \\
& +\exp \left(-\frac{t}{2}\right) \sup _{0 \leq s \leq \frac{t}{2}}\left[\gamma_{2}(|\mu(s)|)+\overline{\mathfrak{k}}|d(s)|\right] \\
& \quad+\sup _{\frac{t}{2} \leq s}\left[\gamma_{2}(|\mu(s)|)+\overline{\mathfrak{k}}|d(s)|\right] .
\end{aligned}
$$

We conclude that, for any closed loop solution, there exists a real number $c$ such that the corresponding $\omega$-limit set is contained in the set $\left\{(\mathcal{X}, \widehat{\mathcal{X}}, y): e^{T} P e=c,|\widehat{\mathcal{X}}|=y=0\right\}$. But, with Assumption DE-D2.2, we know that any solution in this set converges to the origin. So, by following the same arguments as in [12, p.44], we can conclude that we have global attractivity.

\subsection{Discussion}

Compared to the result in [17], in Theorem [4, we are less restrictive in allowing the terms $b_{i}$ to depend also on $\left(y_{2}, \ldots, y_{i}\right)$. But we are more restrictive in not dealing with the (unknown) disturbance terms. In doing so, we can work with a less demanding detectability assumption with replacing an ISS property by a simpler stability property. If we were to cope also with 
these disturbances, as in [17], in the detectability assumption, we would come back to an ISS property and, in the output feedback design, we would replace the propagation of the $L^{2}$-ISS property by the propagation of the ISS property with a gain assignment, a technique introduced in 30.

Also if we compare Theorems 1 and \&, we see that, for the former, the stabilizability assumption SD-S is more restrictive (strict feedback form) but the detectability assumption $\mathrm{SD}-\mathrm{D}$ is weaker. However, if we succeed in proving that the procedure of Freeman and Kokotović proposed in [1] extends to the case of SD-S', then Theorem 11 would give a less restrictive result. Nevertheless, even in this case, Theorem 1 will remain very interesting since the design part of the state feedback is much simpler compared with what can be expected to be obtained from [11].

\section{$5 \quad$ Examples}

Example 1 : Consider the system in $\mathbb{R}^{2}$ :

$$
\left\{\begin{array}{l}
\dot{z}=-z+y^{2} z^{2} \\
\dot{y}=u-z^{2}
\end{array}\right.
$$

We have :

1. With the following reduced order observer :

$$
\hat{z}=w-\frac{y^{3}}{3} \quad, \quad \dot{w}=-w+\frac{y^{3}}{3}+y^{2} u
$$

we obtain :

$$
\overbrace{\frac{1}{2}(\hat{z}-z)^{2}+\frac{1}{4}(\hat{z}-z)^{4}}
$$

$$
=-(\hat{z}-z)^{2}-(\hat{z}-z)^{4} \text {. }
$$

To follow the dynamics error approach, we have to write the dynamics of $\hat{z}$ and $y$ in such a way that the corresponding correction term is an $L^{2}$ function along the solutions. To do this here we decompose $\dot{\hat{z}}$ and $\dot{y}$ as :

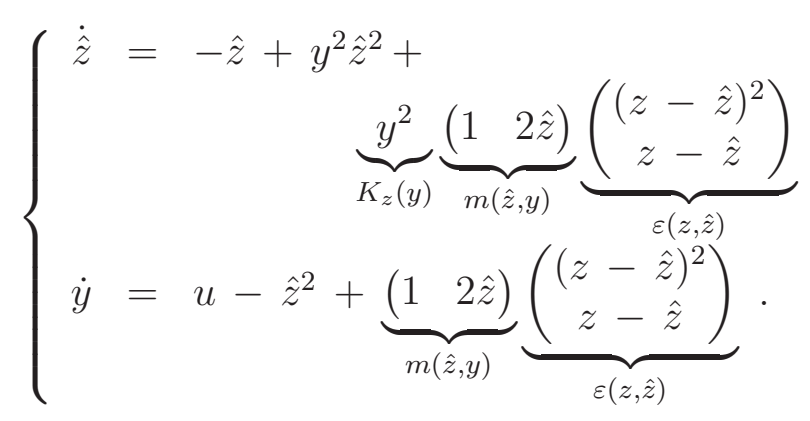

According to the discussion following (30), the correction term is identified as being $\varepsilon$. With (45), we see it is an $L^{2}$ function along the solutions. This proves that the (modified) detectability assumption DE-S2 of the dynamics error approach is satisfied. 
2. For any function $\phi_{z}$, any $z>2$, and $d_{z}$, we have, for all $d_{u} \geq 1$,

$$
\dot{z}=-z+\left(\phi_{z}\left(z+d_{z}\right)\right)^{2} z^{2}+d_{u} z^{2} \geq \frac{1}{2} z^{2} .
$$

Hence the stabilizability assumptions SD-S and SD-S' are not satisfied. Hence only the dynamics error approach can be considered.

3. With $y=\phi_{z}(\hat{z})=0$, and $V_{z}(\hat{z})=\frac{1}{2} \hat{z}^{2}$, we have :

$$
\begin{aligned}
\frac{\partial V_{z}}{\partial z}(\hat{z})\left[-\hat{z}+y^{2} \hat{z}^{2}+y^{2}\left(\begin{array}{ll}
1 & 2 \hat{z}
\end{array}\right)\left(\begin{array}{l}
d_{1} \\
d_{2}
\end{array}\right)\right] & \\
& =-2 V_{z}(\hat{z}) \quad \forall\left(\hat{z}, d_{1}, d_{2}\right) .
\end{aligned}
$$

So the (modified) stabilizability assumptions DE-S2 of the dynamics error approach is satisfied.

From the above and by applying Lemma 1, we can conclude that the output feedback :

$$
\left\{\begin{aligned}
\dot{w} & =-w+\frac{y^{3}}{3}+y^{2} u \\
\hat{z} & =w-\frac{y^{3}}{3} \\
u & =-y+\hat{z}^{2}-y \hat{z}^{3}-\left(y+y^{3} \hat{z}^{2}\right)\left(1+4 \hat{z}^{2}\right)
\end{aligned}\right.
$$

is globally asymptotically stabilizing.

Example 2 : Consider the system (see [7, Example 2]) :

$$
\left\{\begin{array}{l}
\dot{z}=3 z+2 z^{3}+y, \\
\dot{y}=z+z^{3}+u .
\end{array}\right.
$$

We have :

1. From the inequality :

$$
\operatorname{sign}\left(e_{z}\right)\left(\frac{d}{d z}\left[3 z+2 z^{3}\right]-4 \frac{d}{d z}\left[z+z^{3}\right]\right) e_{z}
$$

$$
\leq-\left|\frac{d}{d z}\left[z+z^{3}\right]\right|\left|e_{z}\right|,
$$

we conclude that the inequalities (8) and (33) are satisfied with $P=1, v=0$ and $K=4$. Hence the detectability assumptions $S D-D$ of the state disturbance approach and the detectability assumption DE-D1 of the dynamics error approach in the $L^{1}$-case are satisfied.

2. The $z$ dynamics are in strict feedback form, thus the stabilizability assumption $S D$-S of the state disturbance approach is satisfied. Also with :

$\phi_{z}(\hat{z})=-4 \hat{z}-2 \hat{z}^{3}$

$$
V_{z}(\hat{z})=\sqrt{1+\hat{z}^{2}}-1,
$$

we get : 


$$
\frac{\partial V}{\partial z}(\hat{z})\left[3 \hat{z}+2 \hat{z}^{3}+\phi_{z}(\hat{z})+d\right]
$$

$$
\leq-\frac{\hat{z}^{2}}{\sqrt{1+\hat{z}^{2}}}+|d| .
$$

This proves that the stabilizability assumption DE-S1 of the dynamics error approach is satisfied also.

From the above and by applying Lemma 2 for instance, we can conclude that the output feedback :

$$
\left\{\begin{array}{l}
\dot{w}=-\hat{z}-2 \hat{z}^{3}-4 u+y \\
\hat{z}=w+4 y \\
u=-\hat{z}-\hat{z}^{3}-\left(4+6 \hat{z}^{2}\right)\left(3 \hat{z}+2 \hat{z}^{3}+y\right) \\
+r(y, \hat{z})\left(-4 \hat{z}-2 \hat{z}^{3}-y-24\left|1+\hat{z}^{3}\right| \frac{\hat{z}}{\sqrt{1+\hat{z}^{2}}}\right) .
\end{array}\right.
$$

where $r(y, \hat{z})=1+\frac{1}{2}\left(y+4 \hat{z}+2 \hat{z}^{3}\right)^{2}$ is globally asymptotically stabilizing.

Example 3 : Consider the system (see [29]) :

$$
\left\{\begin{aligned}
\dot{x}_{1} & =x_{2}+u \\
\dot{x}_{2} & =f\left(x_{1}\right)+x_{3}-u, \\
\dot{x}_{3} & =-f\left(x_{1}\right) \\
y & =x_{1}
\end{aligned}\right.
$$

where $f$ is a $C^{3}$ function such that $f(0)=0$ and $f^{\prime}(0) \neq 0$.

This system (47) is of the form studied by Marino and Tomei in 21]. But it is proved in [3] that the assumptions of [21] are not satisfied if $f$ possesses another zero not at the origin.

To be within the framework of this paper, we rewrite the dynamics of (47) as :

$$
\left\{\begin{array}{rl}
\dot{z}_{1} & =z_{1}-y \\
\dot{z}_{2} & =-f(y) \\
\dot{y} & =z_{1}-z_{2}-y+u
\end{array} .\right.
$$

We have

1. From the inequalities:

$$
\operatorname{sign}\left(2 e_{z_{1}}-3 e_{z_{2}}\right)(2-3)
$$

$$
\begin{aligned}
& {\left[\left(\begin{array}{ll}
1 & 0 \\
0 & 0
\end{array}\right)-\left(\begin{array}{l}
6 \\
2
\end{array}\right)\left(\begin{array}{ll}
1 & -1
\end{array}\right)\right]} \\
& \qquad\left(\begin{array}{l}
e_{z_{1}} \\
e_{z_{2}}
\end{array}\right)=-2\left|2 e_{z_{1}}-3 e_{z_{2}}\right|
\end{aligned}
$$

$\operatorname{sign}\left(e_{z_{1}}-2 e_{z_{2}}\right)\left(\begin{array}{ll}1 & -2\end{array}\right)$

$$
\begin{aligned}
{\left[\left(\begin{array}{ll}
1 & 0 \\
0 & 0
\end{array}\right)-\left(\begin{array}{l}
6 \\
2
\end{array}\right)\left(\begin{array}{ll}
1 & -1
\end{array}\right)\right] } \\
\qquad\left(\begin{array}{l}
e_{z_{1}} \\
e_{z_{2}}
\end{array}\right)=-\left|e_{z_{1}}-2 e_{z_{2}}\right|
\end{aligned}
$$




$$
\left|e_{z_{1}}-e_{z_{2}}\right| \leq\left|2 e_{z_{1}}-3 e_{z_{2}}\right|+\left|2 e_{z_{2}}-e_{z_{1}}\right|
$$

we conclude that the inequality (33) is satisfied with $P=0, v_{1}=\left(\begin{array}{r}2 \\ -3\end{array}\right), v_{2}=\left(\begin{array}{r}1 \\ -2\end{array}\right)$ and $K=\left(\begin{array}{l}6 \\ 2\end{array}\right)$. Hence the detectability assumption DE-D1 of the dynamics error approach in the $L^{1}$-case is satisfied.

2. Let $a, \sigma$ and $\delta$ be the real number and functions defined as :

$$
\begin{aligned}
& a=\frac{1}{2\left(2 \max _{|s| \leq 3}\left|f^{\prime \prime}(s)\right|\right)^{2}}, \\
& \sigma\left(z_{1}\right)=\max _{|s| \leq 1}\left|f^{\prime \prime}\left(2 z_{1}+s\right)\right|, \\
& \delta\left(z_{1}, s\right)=\sqrt{2 a}\left|f\left(2 z_{1}\right)+f^{\prime}\left(2 z_{1}\right) s-f\left(2 z_{1}+s\right)\right| .
\end{aligned}
$$

Let also $k$ be a $C^{2}$, positive definite and proper functions whose derivative is non decreasing and satisfies :

$$
\left|k^{\prime}\left(z_{1}\right)\right| \geq \max \left\{\sqrt{2 a}\left|z_{1}\right| \sigma\left(z_{1}\right), \frac{f\left(2 z_{1}\right)}{\sqrt{2 a}}\right\} .
$$

Since $f$ is $C^{2}$, we have $\forall|s| \leq 1$ :

$$
\begin{aligned}
\delta\left(z_{1}, s\right) \leq \frac{\sqrt{2 a} \sigma\left(z_{1}\right) s^{2}}{2} & \leq \frac{z_{1} k^{\prime}\left(z_{1}\right)}{2} \\
& \quad \forall 1 \leq\left|z_{1}\right| \\
\leq \frac{s^{2}}{2} & \forall\left|z_{1}\right| \leq 1 .
\end{aligned}
$$

Now, inspired by a forwarding technique (see [25]), we consider $V_{0 z}$, the $C^{2}$, positive definite and proper function defined as :

$$
V_{0 z}\left(z_{1}, z_{2}\right)=k\left(z_{1}\right)+2\left(\sqrt{1+\frac{a}{2} \zeta_{2}^{2}}-1\right)
$$

where :

$$
\zeta_{2}=\left(z_{2}-\int_{0}^{z_{1}} \frac{f(2 s)}{s} d s\right)
$$

With (49) and :

$$
y=\phi_{z}\left(z_{1}, z_{2}\right)=2 z_{1}+s
$$

where $s$ in $(-1,1)$ is

$s=\frac{2}{\pi} \arctan \left(k^{\prime}\left(z_{1}\right)\right.$

$$
\left.-\frac{a \zeta_{2}}{\sqrt{1+\frac{a}{2} \zeta_{2}^{2}}}\left(-f^{\prime}\left(2 z_{1}\right)+\frac{f\left(2 z_{1}\right)}{z_{1}}\right)\right),
$$

we get :

$$
\begin{aligned}
\overparen{V_{0 z}\left(z_{1}, z_{2}\right)} & \leq-z_{1} k^{\prime}\left(z_{1}\right)-s^{2}+\delta\left(z_{1}, s\right) \\
& \leq-\frac{z_{1} k^{\prime}\left(z_{1}\right)+s^{2}}{2}
\end{aligned}
$$


Also, with (48), we have :

$$
\begin{aligned}
\left|\frac{\partial V_{0 z}}{\partial z_{2}}\left(z_{1}, z_{2}\right)\right| \leq \sqrt{2 a}, \quad \forall\left(z_{1}, z_{2}\right) & \\
\left|\frac{\partial V_{0 z}}{\partial z_{1}}\left(z_{1}, z_{2}\right)\right| \leq & \left|k^{\prime}\left(z_{1}\right)\right|+\sqrt{2 a}\left|\frac{f\left(2 z_{1}\right)}{z_{1}}\right| \\
& \leq 2\left|k^{\prime}\left(z_{1}\right)\right| \\
& \leq b,
\end{aligned}
$$$$
\begin{aligned}
& \forall\left(z_{1}, z_{2}\right): 1 \leq\left|z_{1}\right| \\
& \forall\left(z_{1}, z_{2}\right):\left|z_{1}\right| \leq 1
\end{aligned}
$$

where $b=\max _{\left|z_{1}\right| \leq 1}\left\{\left|k^{\prime}\left(z_{1}\right)\right|+\sqrt{2 a} \frac{f\left(2 z_{1}\right)}{z_{1}}\right\}$. Let $\ell: \mathbb{R}_{+} \rightarrow \mathbb{R}_{+}$be a $C^{2}$ and proper function which is zero at zero, has a strictly positive derivative and satisfies :

$$
\ell\left(k\left(z_{1}\right)\right)=\left|z_{1}\right| \quad \forall z_{1}: 1 \leq\left|z_{1}\right| .
$$

So finally we define a function $V_{z}$ as :

$$
V_{z}\left(z_{1}, z_{2}\right)=\ell\left(V_{0 z}\left(z_{1}, z_{2}\right)\right)
$$

It is $C^{2}$, positive definite and proper with a bounded gradient. For instance, $\ell^{\prime}$ being non increasing on $[k(1),+\infty)$, we have :

$$
\begin{array}{ll}
\left|\frac{\partial V_{z}}{\partial z_{1}}\left(z_{1}, z_{2}\right)\right| \leq 2 \ell^{\prime}\left(V_{0 z}\left(z_{1}, z_{2}\right)\right) k^{\prime}\left(z_{1}\right) & \\
\quad \leq 2 \ell^{\prime}\left(k\left(z_{1}\right)\right) k^{\prime}\left(z_{1}\right) \leq 2 & \forall\left(z_{1}, z_{2}\right): 1 \leq\left|z_{1}\right| \\
\quad \leq b \ell^{\prime}\left(V_{0 z}\left(z_{1}, z_{2}\right)\right) \leq b \max _{\left|z_{1}\right| \leq 1} \ell^{\prime}\left(k\left(z_{1}\right)\right) & \\
& \forall\left(z_{1}, z_{2}\right):\left|z_{1}\right| \leq 1 .
\end{array}
$$

Hence, with $V_{z}$ and $\phi_{z}$ defined above, the stabilizability assumption DE-S1 of the dynamics error approach is satisfied.

With Theorem 3 , we conclude that there exists a globally asymptotically stabilizing output feedback.

Example 4 : Consider the system :

$$
\left\{\begin{aligned}
\dot{z} & =z^{2}+y \\
\dot{y} & =y_{2}+z^{3}, \\
\dot{y}_{2} & =u+2 z^{3}+z^{2}+2 z .
\end{aligned}\right.
$$

We have :

1. Suppose there exists a reduced order observer which satisfies the detectability assumption DE-D2 of the dynamics error approach in the $L^{2}$-case. Then we have 3 real numbers $(p, q, r)$ and two functions $K_{z}$ and $K_{2}$ such that, for each $\left(e_{z}, e_{2}, z, y\right)$, we get :

$$
\begin{aligned}
\left(\begin{array}{ll}
e_{z} & e_{2}
\end{array}\right)\left(\begin{array}{ll}
p & q \\
q & r
\end{array}\right) & {\left[\left(\begin{array}{cc}
2 z & 0 \\
6 z^{2}+2 z+2 & 0
\end{array}\right)-\left(\begin{array}{c}
K_{z}(y) \\
K_{2}(y)
\end{array}\right)\left(\begin{array}{ll}
3 z^{2} & 1
\end{array}\right)\right] }
\end{aligned}
$$




$$
\begin{array}{r}
\left(\begin{array}{l}
e_{z} \\
e_{2}
\end{array}\right) \\
\leq-\left(\left(\begin{array}{ll}
3 z^{2} & 1
\end{array}\right)\left(\begin{array}{l}
e_{z} \\
e_{2}
\end{array}\right)\right)^{2} .
\end{array}
$$

When $e_{2}=0$, this gives :

$9 z^{4}+q\left(6 z^{2}+2 z+2-3 K_{2}(y) z^{2}\right)$

$$
+p\left(2 z-3 K_{z}(y) z^{2}\right) \leq 0 \quad \forall(z, y) .
$$

For any given $y$, the left hand side of this inequality goes to $+\infty$ when $z$ goes to infinity, which is impossible. This proves that the detectability assumption DE-D2 of the dynamics error approach in the $L^{2}$-case cannot be satisfied.

2. From the following inequalities :

$\operatorname{sign}\left(e_{z}\right)\left(\begin{array}{ll}1 & 0\end{array}\right)$

$$
\begin{aligned}
& {\left[\left(\begin{array}{cc}
2 z & 0 \\
6 z^{2}+2 z+2 & 0
\end{array}\right)\right.}\left.-\left(\begin{array}{l}
1 \\
3
\end{array}\right)\left(\begin{array}{cc}
3 z^{2} & 1
\end{array}\right)\right] \\
& \qquad\left(\begin{array}{c}
e_{z} \\
e_{2}
\end{array}\right) \\
&=-\left(3 z^{2}-2 z+1\right)\left|e_{z}\right|+\operatorname{sign}\left(e_{z}\right)\left(e_{z}-e_{2}\right) \\
& \leq-\left(3 z^{2}-2 z+1\right)\left|e_{z}\right|+\left|e_{z}-e_{2}\right|
\end{aligned}
$$

$\operatorname{sign}\left(e_{z}-e_{2}\right)\left(\begin{array}{ll}1 & -1\end{array}\right)$

$$
\begin{gathered}
{\left[\left(\begin{array}{cc}
2 z & 0 \\
6 z^{2}+2 z+2 & 0
\end{array}\right)-\left(\begin{array}{l}
1 \\
3
\end{array}\right)\left(\begin{array}{ll}
3 z^{2} & 1
\end{array}\right)\right]} \\
\left(\begin{array}{l}
e_{z} \\
e_{2}
\end{array}\right)
\end{gathered}
$$

$\frac{1}{3}\left[3 z^{2} e_{z}+e_{2}\left|\leq \frac{1}{3}\left(3 z^{2}+1\right)\right| e_{z}\left|+\frac{1}{3}\right| e_{z}-e_{2} \mid\right.$

$$
\leq\left(3 z^{2}-2 z+1\right)\left|e_{z}\right|+\left|e_{z}-e_{2}\right| \text {, }
$$

we conclude that the inequality (B3) is satisfied with $P=0, v_{1}=\left(\begin{array}{l}1 \\ 0\end{array}\right), v_{2}=\left(\begin{array}{r}1 \\ -1\end{array}\right)$ and $K=\left(\begin{array}{l}1 \\ 3\end{array}\right)$. Hence the detectability assumption DE-D1 of the dynamics error approach in the $L^{1}$-case is satisfied.

3. With $V_{z}(\hat{z})=\sqrt{1+\hat{z}^{2}}-1$ and $\phi_{z}(\hat{z})=-\hat{z}^{2}-\hat{z}$ we get :

$$
\frac{\partial V_{z}}{\partial \hat{z}}(\hat{z})\left[\hat{z}^{2}+\phi_{z}(\hat{z})+d\right] \leq \frac{-\hat{z}^{2}}{\sqrt{1+\hat{z}^{2}}}+|d| \text {. }
$$


It follows that the stabilizability assumption DE-S1 of the dynamics error approach in the $L^{1}$-case is satisfied.

With Theorem 3, we conclude that there exists a globally asymptotically stabilizing output feedback.

\section{Conclusion}

We have investigated the problem of global asymptotic stabilization by output feedback for systems whose dynamics admit a strict normal form. By rephrasing and formalizing already known approaches we have been able to introduce several sets of assumptions that allow us to design an output feedback. As in the results given by Karagiannis, Jiang, Ortega and Astolfi in [17], and Marino and Tomei in [21] no minimum-phase assumptions are required but instead we ask for stabilizability by a state control of the inverse dynamics with various robustness properties depending on what can be achieved with a reduced order observer.

\section{Appendix :}

\section{Propagation of the $L^{p}$-ISS property through a chain of integrators}

We consider a system in the form :

$$
\left\{\begin{array}{l}
\dot{x_{1}}=f\left(x_{1}, x_{2}\right)+K_{1}\left(x_{1}, x_{2}\right) d_{1} \\
\dot{x}_{2}=a\left(x_{1}, x_{2}\right) u+b\left(x_{1}, x_{2}\right)+K_{2}\left(x_{1}, x_{2}\right) d_{2}
\end{array}\right.
$$

where $x_{1}$ is in $\mathbb{R}^{n_{1}}, x_{2}$ is in $\mathbb{R}, u$ is in $\mathbb{R}, d_{1}$ is in $\mathbb{R}^{n_{1}}, d_{2}$ in $\mathbb{R}, a\left(x_{1}, x_{2}\right)$ is strictly positive and the functions $f, K_{1}, a, b$ and $K_{2}$ are as many times differentiable as needed below.

Lemma 1 ( $L^{2}$-ISS propagation) Suppose there exist a $C^{q+1}$, positive definite and proper function $V_{1}: \mathbb{R}^{n_{1}} \rightarrow \mathbb{R}_{+}$, a $C^{q+1}$ function $\phi_{1}: \mathbb{R}^{n_{1}} \rightarrow \mathbb{R}$, and a positive definite continuous function $\alpha_{1}: \mathbb{R}^{n_{1}} \rightarrow \mathbb{R}_{+}$such that, along the solutions of (51), we have :

$\overparen{V_{1}\left(x_{1}\right)} \leq-\alpha_{1}\left(x_{1}\right)+\left|d_{1}\right|^{2}$

$$
\forall\left(x_{1}, x_{2}, d_{1}\right): x_{2}=\phi_{1}\left(x_{1}\right) .
$$

Then, there exists a $C^{q}$, positive definite and proper function $V_{2}: \mathbb{R}^{n_{1}+1} \rightarrow \mathbb{R}_{+}$, a $C^{q}$ function $\phi_{2}: \mathbb{R}^{n_{1}+1} \rightarrow \mathbb{R}$, and a positive definite continuous function $\alpha_{2}: \mathbb{R}^{n_{1}+1} \rightarrow \mathbb{R}_{+}$such that, along the solutions of (51), we get :

$\overparen{V_{2}\left(x_{1}, x_{2}\right)} \leq-\alpha_{2}\left(x_{1}, x_{2}\right)+\left|d_{1}\right|^{2}+\left|d_{2}\right|^{2}$

$$
\forall\left(x_{1}, x_{2}, d_{1}, d_{2}, u\right): u=\phi_{2}\left(x_{1}, x_{2}\right)
$$

This result is well known. See [15, 30, 1] for a proof. 
Lemma 2 ( $L^{1}$-ISS propagation ) Suppose the function $K_{1}$ does not depend on $x_{2}$ and there exist a continuous function $M: \mathbb{R}^{n_{1}} \rightarrow \mathbb{R}_{+}$, a $C^{q+1}$, positive definite and proper function $V_{1}: \mathbb{R}^{n_{1}} \rightarrow \mathbb{R}_{+}$, a $C^{q+1}$ function $\phi_{1}: \mathbb{R}^{n_{1}} \rightarrow \mathbb{R}$, and a positive definite continuous function $\alpha_{1}: \mathbb{R}^{n_{1}} \rightarrow \mathbb{R}_{+}$satisfying :

$$
\left|K_{2}\left(x_{1}, x_{2}\right)\right| \leq M\left(x_{1}\right)\left(1+\left|x_{2}\right|\right) .
$$

and, along the solutions of (51),

$\overparen{V_{1}\left(x_{1}\right)} \leq-\alpha_{1}\left(x_{1}\right)+\left|d_{1}\right|$

$$
\forall\left(x_{1}, x_{2}, d_{1}\right): x_{2}=\phi_{1}\left(x_{1}\right) .
$$

Then, there exist a $C^{q}$, positive definite and proper function $V_{2}: \mathbb{R}^{n_{1}+1} \rightarrow \mathbb{R}_{+}$, a $C^{q}$ function $\phi_{2}: \mathbb{R}^{n_{1}+1} \rightarrow \mathbb{R}$, and a positive definite continuous function $\alpha_{2}: \mathbb{R}^{n_{1}+1} \rightarrow \mathbb{R}_{+}$such that, along the solutions of (51), we get:

$\overparen{V_{2}\left(x_{1}, x_{2}\right)} \leq-\alpha_{2}\left(x_{1}, x_{2}\right)+\left|d_{1}\right|+\left|d_{2}\right|$

$$
\forall\left(x_{1}, x_{2}, d_{1}, d_{2}, u\right): u=\phi_{2}\left(x_{1}, x_{2}\right)
$$

Proof : We follow here a suggestion of Frederic Mazenc who used a very similar argument in his dissertation [24, (2.412)].

As the function $V_{1}$ is proper, we can find a $C^{q}$ and increasing function $k^{\prime}: \mathbb{R}_{+} \rightarrow \mathbb{R}_{+}$ satisfying for all $x_{1}$ in $\mathbb{R}^{n_{1}}$ :

$k^{\prime}\left(V\left(x_{1}\right)\right) \geq \max \left\{1,\left|\frac{\partial \phi_{1}}{\partial x_{1}}\left(x_{1}\right)\right|\left|K_{1}\left(x_{1}\right)\right|\right.$,

$$
\left.\frac{1}{2} M\left(x_{1}\right)\left(3+\left|\phi_{1}\left(x_{1}\right)\right|\right)\right\} .
$$

Let $W: \mathbb{R}^{n_{1}} \times \mathbb{R} \rightarrow \mathbb{R}_{+}$be the $C^{q}$, positive definite and proper function defined as :

$W\left(x_{1}, x_{2}\right)=k\left(V_{1}\left(x_{1}\right)\right)$

$$
+\log \left(1+\frac{1}{2}\left(x_{2}-\phi\left(x_{1}\right)\right)^{2}\right)
$$

where $k$ is the $C^{q+1}$ and proper function defined as :

$$
k(s)=\int_{0}^{s} k^{\prime}(u) d u \quad, \quad \forall s \in \mathbb{R}_{+} .
$$

By differentiating $W$ along the solutions of (51), we get :

$$
\begin{aligned}
\overparen{W\left(x_{1}, x_{2}\right)} \leq-k^{\prime}\left(V_{1}\left(x_{1}\right)\right) \alpha_{1}\left(x_{1}\right) & \\
& \quad+m_{1}\left(x_{1}, x_{2}\right)\left|d_{1}\right|+m_{2}\left(x_{1}, x_{2}\right)\left|d_{2}\right| \\
& \quad+\left(x_{2}-\phi_{1}\left(x_{1}\right)\right)\left[p\left(x_{1}, x_{2}\right)+q\left(x_{1}, x_{2}\right) u\right]
\end{aligned}
$$

where $m_{1}: \mathbb{R}^{n_{1}} \times \mathbb{R}$ and $m_{2}: \mathbb{R}^{n_{1}} \times \mathbb{R}$ are continuous functions, and $p: \mathbb{R}^{n_{1}} \times \mathbb{R}$ is a $C^{q}$ function defined as : 


$$
\begin{gathered}
m_{1}\left(x_{1}, x_{2}\right)=k^{\prime}\left(V_{1}\left(x_{1}\right)\right)+ \\
m_{2}\left(x_{1}, x_{2}\right)=\frac{\left|x_{2}-\phi_{1}\left(x_{1}\right)\right|\left|K_{2}\left(x_{1}, x_{2}\right)\right|}{1+\frac{1}{2}\left(x_{2}-\phi_{1}\left(x_{1}\right)\right)^{2}} \\
p\left(x_{1}, x_{2}\right)=\frac{b\left(x_{1}, x_{2}\right)-\frac{\partial \phi_{1}}{\partial x_{1}}\left(x_{1}\right) f\left(x_{1}, x_{2}\right)}{1+\frac{1}{2}\left(x_{2}-\phi_{1}\left(x_{1}\right)\right)^{2}}+ \\
k^{\prime}\left(V_{1}\left(x_{1}\right)\right) \frac{\partial V_{1}}{\partial x_{1}}\left(x_{1}\right) . \\
q\left(x_{1}, x_{2}\right)=\frac{a\left(x_{1}, x_{2}\right)}{1+\frac{1}{2}\left(x_{2}-\phi_{1}\left(x_{1}\right)\right)^{2}},
\end{gathered}
$$$$
\frac{\left|x_{2}-\phi_{1}\left(x_{1}\right)\right|\left|\frac{\partial \phi_{1}}{\partial x_{1}}\left(x_{1}\right)\right|\left|K_{1}\left(x_{1}\right)\right|}{1+\frac{1}{2}\left(x_{2}-\phi_{1}\left(x_{1}\right)\right)^{2}}
$$

$$
\int_{0}^{1} \frac{\partial f}{\partial x_{2}}\left(x_{1}, \phi_{1}\left(x_{1}\right)+s\left(x_{2}-\phi_{1}\left(x_{1}\right)\right)\right) d s
$$

where $q$ is non zero from the same assumption on $a$. So let $\phi_{2}$ be the following $C^{q}$ function :

$$
\phi_{2}\left(x_{1}, x_{2}\right)=\frac{1}{q\left(x_{1}, x_{2}\right)}\left(\phi_{1}\left(x_{1}\right)-x_{2}-p\left(x_{1}, x_{2}\right)\right) .
$$

When $u=\phi_{2}\left(x_{1}, x_{2}\right)$, we obtain, along the solutions of (51),

$$
\begin{aligned}
& \overparen{W\left(x_{1}, x_{2}\right)} \leq-k^{\prime}\left(V_{1}\left(x_{1}\right)\right) \alpha_{1}\left(x_{1}\right)-\left(x_{2}-\phi_{1}\left(x_{1}\right)\right)^{2} \\
& +m_{1}\left(x_{1}, x_{2}\right)\left|d_{1}\right|+m_{2}\left(x_{1}, x_{2}\right)\left|d_{2}\right| .
\end{aligned}
$$

Furthermore, from (53), (56) and (57), and from the fact that $k^{\prime}$ is an increasing function, we get :

$$
\begin{aligned}
m_{1}\left(x_{1}, x_{2}\right) & \leq 2 k^{\prime}\left(V_{1}\left(x_{1}\right)\right) \leq 2 k^{\prime}\left(k^{-1}\left(W\left(x_{1}, x_{2}\right)\right)\right) \\
m_{2}\left(x_{1}, x_{2}\right) & \leq \frac{\left|x_{2}-\phi_{1}\left(x_{1}\right)\right|}{1+\frac{1}{2}\left(x_{2}-\phi_{1}\left(x_{1}\right)\right)^{2}} M\left(x_{1}\right) \\
& \leq M\left(x_{1}\right)\left(3+\left|\phi_{1}\left(x_{1}\right)\right|\right) \leq 2 k^{\prime}\left(V_{1}\left(x_{1}\right)\right) \\
& \leq 2 k^{\prime}\left(k^{-1}\left(W\left(x_{1}, x_{2}\right)\right)\right) .
\end{aligned}
$$

Thus, we have :

$\overparen{W\left(x_{1}, x_{2}\right)} \leq-k^{\prime}\left(V_{1}\left(x_{1}\right)\right) \alpha_{1}\left(x_{1}\right)-\left(x_{2}-\phi_{1}\left(x_{1}\right)\right)^{2}$

$$
+2 k^{\prime}\left(k^{-1}\left(W\left(x_{1}, x_{2}\right)\right)\right)\left(\left|d_{1}\right|+\left|d_{2}\right|\right) .
$$

By taking, $V_{2}\left(x_{1}, x_{2}\right)=\ell\left(W\left(x_{1}, x_{2}\right)\right)$ where $\ell$ is the $C^{q}$ and proper function defined as $\ell(s)=\frac{1}{2} k^{-1}(s)$, we obtain finally :

$$
\begin{aligned}
\overparen{V_{2}\left(x_{1}, x_{2}\right)} \leq & -\ell^{\prime}\left(W\left(x_{1}, x_{2}\right)\right) \\
& {\left[k^{\prime}\left(V_{1}\left(x_{1}\right)\right) \alpha_{1}\left(x_{1}\right)+\left(x_{2}-\phi_{1}\left(x_{1}\right)\right)^{2}\right] }
\end{aligned}
$$$$
+\left|d_{1}\right|+\left|d_{2}\right|
$$ 


\section{References}

[1] V. Andrieu, Bouclage de sortie et observateur. Mémoire de thèse, ENSMP. December, (2005), http://pastel.paristech.org/bib/archive/00001530/.

[2] V. Andrieu, L. Praly, A. Astolfi, Nonlinear output feedback design via domination and generalized weighted homogeneity. Proc. of the 45nd IEEE CDC, December, (2006).

[3] V. Andrieu, L. Praly, Global asymptotic stabilization by output feedback for some non minimum phase non linear systems. Proc. of the 44 th IEEE CDC, December, (2005).

[4] M. Arcak, D. Angeli, E. Sontag, A Unifying Integral ISS Framework for Stability of Nonlinear Cascades. SIAM J.. on Opt. and Cont., No. 40, pp. 1888-1904, (2002).

[5] M. Arcak, P. Kokotović, Nonlinear observers : a circle criterion design and robustness analysis. Automatica, No. 37, 1923-1930, (2001).

[6] M. Arcak, P. Kokotović, Observer-Based Control of Systems with Slope-Restricted Nonlinearities. IEEE Trans. on Automat. Cont., Vol. 46, No. 7, July (2001).

[7] M. Arcak, Certainty-Equivalence Output-Feedback Design with Circle-Criterion Observers. IEEE Trans. on Automat. Cont., Vol. 50, No. 6, June (2005).

[8] A. Atassi, H. Khalil, A separation principle for the stabilization of a class of nonlinear systems, IEEE Transactions on Automatic Control, Vol. 44, No. 9, September 1999.

[9] C. Byrnes, A. Isidori, Asymptotic stabilization of minimum phase nonlinear systems. IEEE Trans. on Automat. Cont., Vol. 36, No. 10, October, (1991).

[10] C. Ebenbauer, T.Raff, and F. Allgöwer, A simple separation result for nonlinear control systems. Proc. 16th IFAC World Congress, Prague, Czech Republic, (2005).

[11] R.A. Freeman, P.V. Kokotović, Global robustness of nonlinear systems to state measurement disturbances. Proc. of the 32nd IEEE CDC, December, (1993).

[12] A. Isidori, Nonlinear Control Systems II. Springer Verlag, (1999).

[13] G. Kaliora, A. Astolfi, L.Praly, Norm estimators and global output feedback stabilization of nonlinear systems with ISS inverse dynamics. Proc. of the $43 \mathrm{rd}$ IEEE CDC, December, (2004).

[14] I. Kanellakopoulos, M. Krstić, P. Kokotović, Interlaced controller-observer design for adaptive nonlinear control. Proc. of the 1992 American Control Conference, June 1992

[15] I. Kanellakopoulos, P. V. Kokotović, and A. S. Morse. A toolkit for nonlinear feedback design. Systems $\&$ Control Letters, No. 18, 83-92, (1992).

[16] I. Kanellakopoulos, P. V. Kokotović, and A. S. Morse. Adaptive Output-Feedback Control of a class of Nonlinear Systems. Proc. of the 30th IEEE CDC, December (1991).

[17] D.Karagiannis, Z-P. Jiang, R. Ortega, A. Astolfi, Output-feedback stabilization of a class of uncertain non-minimum-phase nonlinear systems. Automatica, No. 41(9), 1609-1615, (2005). 
[18] P. Krishnamurthy and F. Khorrami, Dynamic high-gain scaling : State and output feedback with application to systems with ISS appended dynamics driven by all states. IEEE Trans. on Automat. Cont., Vol. 49, No. 12, December (2004).

[19] P. Krishnamurthy and F. Khorrami, On uniform solvability of parameter-dependent lyapunov inequalities and applications to various problems. SIAM J. Control Optim., Vol. 45, No. 4,pp. 11471164, 2006.

[20] M. Krstić, I. Kanellakopoulos, P. Kokotović, Nonlinear and adaptive control design. John Wiley 85 Sons, New York, (1995).

[21] R. Marino, P. Tomei, A class of globally output feedback stabilizable nonlinear nonminimum phase systems. IEEE Trans. on Automat. Cont., Vol. 50, No. 12, December, (2005).

[22] R. Marino, P. Tomei, Global Adaptive Output-Feedback Control of Nonlinear Systems. Proc. of the 30th IEEE CDC, December, (1991).

[23] R. Marino, P. Tomei, Nonlinear control design. Geometric, adaptive, robust. Prentice Hall, (1995).

[24] F. Mazenc, Stabilisation de trajectoires, ajout d'intégration, commandes saturées. Mémoire de thèse, ENSMP. Avril, (1996).

[25] F. Mazenc, L. Praly, Adding integrations, saturated controls and global asymptotic stabilization for feedforward systems, IEEE Transactions on Automatic Control, Vol.41, N. 11, (1996).

[26] A. Polański, On absolute stability analysis by polyhedral Lyapunov functions Automatica, Volume 36, Issue 4, April 2000, Pages 573-578

[27] L. Praly, Lyapunov design of a dynamic output feedback for systems linear in their unmeasured state components. Proc. IFAC NOLCOS'92. Bordeaux, June 1992.

[28] L. Praly, Asymptotic stabilization via output feedback for lower triangular systems with output dependent incremental rate. IEEE Trans. on Automat. Cont., Vol. 48, No. 6, June, (2003).

[29] L. Praly, M. Arcak, A relaxed condition for stability of nonlinear observer-based controllers. Systems \& Control Letters, No. 53, 311-320, (2004).

[30] L. Praly, Z.-P. Jiang, Stabilization by output feedback for systems with ISS inverse dynamics. Systems \& Control Letters, No. 21, 19-33, (1993).

[31] C. Qian, A Homogeneous Domination Approach for Global Output Feedback Stabilization of a Class of Nonlinear Systems, Proc. of the ACC, Portland, (2005).

[32] N. Rouché, P. Habets, M. Laloy, Stability theory by Liapunov's direct methods. Applied mathematical sciences 22. Springer Verlag 1977.

[33] A. Teel, L. Praly, Global stabilizability and observability imply semi-global stabilizability by output feedback, Systems \& Control Letters 22 (1994) 313-325 
[34] B. Yang and W. Lin, Further Results on Output Feedback Stabilization of Uncertain Nonlinear Systems. Int. J. of Robust and Nonlinear Control, Vol. 15, pp. 247-268, (2005). 